\title{
ON ROUTING PROBLEM WITH STARTING POINT OPTIMIZATION ${ }^{1}$
}

\author{
Alexander G. Chentsov ${ }^{a), b), \dagger, \text { Pavel A. Chentsov }}{ }^{b)}$ \\ a) Krasovskii Institute of Mathematics and Mechanics, \\ Ural Branch of the Russian Academy of Sciences, \\ 16 S. Kovalevskaya Str., Ekaterinburg, 620108, Russia \\ b) Ural Federal University, \\ 19 Mira Str., Ekaterinburg, 620002, Russia \\ †chentsov@imm.uran.ru
}

\begin{abstract}
One problem focused on engineering applications is considered. It is assumed that sequential visits to megacities have been implemented. After all visits have been made, it is required to return to the starting point (a more complex dependence on the starting point is also considered). But the last requirement is not strict: some weakening of the return condition is acceptable. Under these assumptions, it is required to optimize the choice of starting point, route, and specific trajectory. The well-known dynamic programming (DP) is used for the solution. But when using DP, significant difficulties arise associated with the dependence of the terminal component of the criterion on the starting point. Starting point enumeration is required. We consider the possibility of reducing the enumeration associated with applied variants of universal (relative to the starting point) dynamic programming. Of course, this approach requires some transformation of the problem.
\end{abstract}

Keywords: Dynamic programming, Precedence conditions, Route.

\section{Introduction}

This study addresses the routing problem with precedence conditions and complicated cost functions. Besides, it is required to implement a return to the neighborhood of the starting point (more general variants are also considered). This condition may be related to the peculiarities of applied problems. We keep in mind the cutting of sheets and dismantling in the nuclear power industry. Of course, the well-known Traveling Salesman Problem (TSP) is a natural prototype for this problem. But in our setting, many new difficulties arise. We will mention just a few related to the TSP investigations; see [1, 7, 9-12, 16-18].

In engineering applications, the problem of visiting megacities often arises. This is due to the possible multivariance of the permutations. So, in the control problem when cutting sheets on CNC machines, these megacities are realized when digitizing the contours of parts; this discretization sampling is required for computer applications. Now let us note the precedence conditions. In addition, in control problems when cutting a sheet, these conditions arise, in particular, for the following reasons: for each part, cutting the inner contours must precede the cutting of the outer contour. Of course, there are other specific reasons for using precedence conditions. Among other restrictions, we note the requirements for thermal conductivity. It is useful to note that these requirements are dynamic in nature: they arise depending on the tasks being performed.

We emphasize the importance of starting point optimization. When dismantling radioactive elements, at a step of moving from the starting point, the performer is under the influence of all

\footnotetext{
${ }^{1}$ This work was supported by the Russian Foundation for Basic Research (projects No. 20-08-00873 (Sections 1-4) and No. 18-07-00637 (Sections 5-7)).
} 
radioactive elements to be dismantled. So this step is very important. Therefore, a rational choice of starting point is important. The requirement to return to a neighborhood of the starting point may be related to reasons for sufficient proximity to the transport tool at the starting point. So, the constraints used arise from the needs of actual applied problems.

Of course, without taking into account the above restrictions, a very difficult extremal problem arises. This problem requires serious formalization and the development of theoretical methods. Therefore, this paper provides a detailed exposition of general mathematical concepts. Besides, we use fairly complex constructions of admissible solutions with a choice of basic components: starting point, route, and trajectory. This hierarchical construction of the solution is important.

We use dynamic programming (DP) as the main solution method. But, in our complete problem, the necessity of enumeration of starting points arises. More precisely, its own DP procedure is required for every starting point (in fact, this procedure is attached to the starting point). Apparently, the enumeration of starting points when employing DP is unavoidable. However, we can try to reduce this enumeration. For this, we use auxiliary DP procedures that are universal relative to the starting point; more precisely, we follow [6]. We construct minorant and majorant procedures using DP. In terms of these procedures realized more simply, we aim at the required reduction of the enumeration. Such a goal is attained by weakening the closed routing problem (but, we use our own method and in more general cases). In this case, we obtain a simpler solution to our complete problem. Also, we use an approach of [6] under more general conditions on movements when visiting megacities. This generalization is related to applied problems (for example, such a construction is required in sheet cutting problems).

\section{General notions and designations}

We use the standard set-theoretical notation (quantifiers and logical connectives), $\varnothing$ stands for the empty set and $\triangleq$ for equality by definition. A family is a set whose elements are also sets. For any objects $x$ and $y$, we denote by $\{x ; y\}$ an unordered pair of $x$ and $y: x \in\{x ; y\}, y \in\{x ; y\}$, and $(z=x) \vee(z=y)$ for every $z \in\{x ; y\}$. If $s$ is an object, then $\{s\} \triangleq\{s ; s\}$ is a singleton containing $s: s \in\{s\}$. Also, sets are objects. For any objects $x$ and $y$, the family $(x, y) \triangleq\{\{x\} ;\{x ; y\}\}$ is the ordered pair (see [14, Ch. II, Sect. 2]) with the first element $x$ and the second element $y$. If $h$ is an ordered pair, then $\operatorname{pr}_{1}(h)$ and $\operatorname{pr}_{2}(h)$ are the first and the second elements of $h$, respectively. If $\mathbf{a}, \mathbf{b}$, and $\mathbf{c}$ are objects, then $(\mathbf{a}, \mathbf{b}, \mathbf{c}) \triangleq((\mathbf{a}, \mathbf{b}), \mathbf{c})$ (see [8, Ch. 1]). If $A, B$, and $C$ are sets, then $A \times B \times C \triangleq(A \times B) \times C$ (see [8, Ch. 1]). For a set $H$, we denote by $\mathcal{P}(H)$ and $\mathcal{P}^{\prime}(H)$ the families of all subsets and all nonempty subsets of $H$, repectively; thus, $\mathcal{P}^{\prime}(H)=\mathcal{P}(H) \backslash\{\varnothing\}$. Denote by $\operatorname{Fin}(H)$ the family of all finite nonempty subsets of the set $H, \operatorname{Fin}(H) \subset \mathcal{P}^{\prime}(H)$. If $H$ is a finite set, then $\operatorname{Fin}(H)=\mathcal{P}^{\prime}(H)$.

If $A, B, C$, and $D$ are nonempty sets, and $g$ is a mapping from $A \times B \times C$ to $D$, then $(x, y) \in A \times B \times C$ for $x \in A \times B$ and $y \in C$, and the value $g(x, y) \in D$ is well defined; we also write this value as $g\left(x_{1}, x_{2}, y\right)$, where $x_{1}=\operatorname{pr}_{1}(x) \in A$ and $x_{2}=\operatorname{pr}_{2}(x) \in B$.

As usual, $\mathbb{R}$ denotes the real line,

$$
\begin{gathered}
\mathbb{R}_{+} \triangleq\{\xi \in \mathbb{R} \mid 0 \leq \xi\}, \quad \mathbb{N} \triangleq\{1 ; 2 ; \ldots\}, \quad \mathbb{N}_{0} \triangleq\{0\} \cup \mathbb{N}=\{0 ; 1 ; 2 ; \ldots\} \in \mathcal{P}^{\prime}\left(\mathbb{R}_{+}\right) \\
\overline{p, q} \triangleq\left\{k \in \mathbb{N}_{0} \mid(p \leq k) \&(k \leq q)\right\} \quad \forall p \in \mathbb{N}_{0} \quad \forall q \in \mathbb{N}_{0} .
\end{gathered}
$$

Of course, $\overline{1,0}=\varnothing$ and $\overline{1, s}=\{k \in \mathbb{N} \mid k \leq s\}$ for $s \in \mathbb{N}$. For a nonempty finite set $K$, denote by $|K| \in \mathbb{N}$ the cardinality of $K$ and by (bi) $[K]$ the set of all bijections from $\overline{1,|K|}$ onto $K$. Let $|\varnothing| \triangleq 0$. For a nonempty set $S$, denote by $\mathcal{R}_{+}[S]$ the set of all nonnegative real-valued functions on $S$. 


\section{The problem setting}

Fix a nonempty set $X$ and a set $X^{0} \in \operatorname{Fin}(X)$. We consider $X$ as a comprehending set and $X^{0}$ as the set of all possible starting points. Let $N \in \mathbb{N}, N \geq 2$. Let

$$
M_{1} \in \operatorname{Fin}(X), \quad \ldots, \quad M_{N} \in \operatorname{Fin}(X) .
$$

We consider the sets from (2.1) as megacities. These megacities are visiting objects. Suppose that

$$
\left(X^{0} \cap M_{j}=\varnothing \quad \forall j \in \overline{1, N}\right) \&\left(M_{p} \cap M_{q}=\varnothing \quad \forall p \in \overline{1, N} \quad \forall q \in \overline{1, N} \backslash\{p\}\right) .
$$

Conditions (2.2) are typical for routing problems. Finally, we fix (nonempty) relations

$$
\mathbb{M}_{1} \in \mathcal{P}^{\prime}\left(M_{1} \times M_{1}\right), \quad \ldots, \quad \mathbb{M}_{N} \in \mathcal{P}^{\prime}\left(M_{N} \times M_{N}\right) .
$$

An ordered pair of $\mathbb{M}_{j}, j \in \overline{1, N}$, defines possible variants of works connected with visiting $M_{j}$. We call these works internal. For every megacity, we introduce arrival points and departure points: $\mathfrak{M}_{j} \triangleq\left\{\operatorname{pr}_{1}(z): z \in \mathbb{M}_{j}\right\}$ and $\mathbf{M}_{j} \triangleq\left\{\operatorname{pr}_{2}(z): z \in \mathbb{M}_{j}\right\}$ for $j \in \overline{1, N}$; of course, $\mathfrak{M}_{j} \in \mathcal{P}^{\prime}\left(M_{j}\right)$ and $\mathbf{M}_{j} \in \mathcal{P}^{\prime}\left(M_{j}\right)$. Moreover, we obtain that

$$
\left(\mathbb{X} \triangleq X^{0} \cup\left(\bigcup_{i=1}^{N} \mathfrak{M}_{i}\right) \in \operatorname{Fin}(X)\right) \&\left(\mathbf{X} \triangleq X^{0} \cup\left(\bigcup_{i=1}^{N} \mathbf{M}_{i}\right) \in \operatorname{Fin}(X)\right) .
$$

Let $\mathfrak{N} \triangleq \mathcal{P}^{\prime}(\overline{1, N})$, and let $\mathfrak{N}^{(j)} \triangleq\{K \in \mathfrak{N} \mid j \in K\}$ for $j \in \overline{1, N}$. We fix $N$ mappings

$$
A_{1}:\left(\mathbf{X} \backslash \mathbf{M}_{1}\right) \times \mathfrak{N}^{(1)} \longrightarrow \mathcal{P}^{\prime}\left(M_{1}\right), \quad \ldots, \quad A_{N}:\left(\mathbf{X} \backslash \mathbf{M}_{N}\right) \times \mathfrak{N}^{(N)} \longrightarrow \mathcal{P}^{\prime}\left(M_{N}\right)
$$

with the following property:

$$
A_{j}(x, K) \cap \mathfrak{M}_{j} \neq \varnothing \quad \forall j \in \overline{1, N} \quad \forall x \in \mathbf{X} \backslash \mathbf{M}_{j} \quad \forall K \in \mathfrak{N}^{(j)} .
$$

The mappings (2.4) are used for constraints representation; (2.5) is a compatibility condition. We note that our construction is similar to [2, Sect. 2]. But our mappings (2.4) are defined on smaller sets as compared with analogous mappings from [2, Sect. 2]. Note that our mappings (2.4) can be extended to analogous mappings from [2] (the corresponding variant was considered in [2, p. 215]). In this extension of our definition, conditions (2.5) turn into [2, (3)]. So, this extension is an unessential operation.

In what follows, $\mathbb{P} \triangleq(\mathrm{bi})[\overline{1, N}]$; elements of $\mathbb{P}$ are complete routes (index permutations). If $\alpha \in \mathbb{P}$, then $\alpha^{-1} \in \mathbb{P}$ is the inverse of $\alpha$ :

$$
\alpha\left(\alpha^{-1}(k)\right)=\alpha^{-1}(\alpha(k))=k \quad \forall k \in \overline{1, N} .
$$

A specific choice of $\alpha \in \mathbb{P}$ may be restricted by precedence conditions. For their introduction, we suppose that a set $\mathbf{K} \in \mathcal{P}(\overline{1, N} \times \overline{1, N})$ is given. Let

$$
\forall \mathbf{K}_{0} \in \mathcal{P}^{\prime}(\mathbf{K}) \quad \exists z_{0} \in \mathbf{K}_{0}: \operatorname{pr}_{1}\left(z_{0}\right) \neq \operatorname{pr}_{2}(z) \quad \forall z \in \mathbf{K}_{0} .
$$

Specific cases of (2.6) were discussed in [3, Ch. 2]. Let

$$
\begin{gathered}
\mathbf{A} \triangleq\{\alpha \\
\left.\in \mathbb{P} \mid \forall t_{1} \in \overline{1, N} \forall t_{2} \in \overline{1, N}\left(\left(\alpha\left(t_{1}\right), \alpha\left(t_{2}\right)\right) \in \mathbf{K}\right) \Longrightarrow\left(t_{1}<t_{2}\right)\right\} \\
=\left\{\alpha \in \mathbb{P} \mid \alpha^{-1}\left(\operatorname{pr}_{1}(z)\right)<\alpha^{-1}\left(\operatorname{pr}_{2}(z)\right) \quad \forall z \in \mathbf{K}\right\} \neq \varnothing
\end{gathered}
$$


be the set of all routes (permutations) admissible by precedence. We consider the following processes:

$$
\begin{aligned}
\left(x \in X^{0}\right) \longrightarrow\left(x_{1}^{(1)} \in A_{\alpha(1)}(x, \overline{1, N}) \rightsquigarrow x_{2}^{(1)} \in \mathbf{M}_{\alpha(1)}\right) \longrightarrow\left(x_{1}^{(2)} \in A_{\alpha(2)}\left(x_{2}^{(1)}, \overline{1, N} \backslash\{\alpha(1)\}\right)\right. \\
\left.\rightsquigarrow x_{2}^{(2)} \in \mathbf{M}_{\alpha(2)}\right) \longrightarrow \cdots \longrightarrow\left(x_{1}^{(N)} \in A_{\alpha(N)}\left(x_{2}^{(N-1)},\{\alpha(N)\}\right) \rightsquigarrow x_{2}^{(N)} \in \mathbf{M}_{\alpha(N)}\right)
\end{aligned}
$$

where $\alpha \in \mathbf{A}$ and $\left(x_{1}^{(j)}, x_{2}^{(j)}\right) \in \mathbb{M}_{\alpha(j)}$ for $j \in \overline{1, N}$ (here, we suppose that the number $N$ is sufficiently great). From (2.7), it is obvious that a trajectory coordinated with the route $\alpha$ is used. Let us introduce the corresponding definition. Let $\mathbb{Z}$ be the set of all mappings from $\overline{0, N}$ to $\mathbb{X} \times \mathbf{X}$. So, elements of $\mathbb{Z}$ are tuples

$$
\left(z_{i}\right)_{i \in \overline{0, N}}: \overline{0, N} \longrightarrow \mathbb{X} \times \mathbf{X}
$$

and only they. If $x \in X^{0}$ and $\alpha \in \mathbb{P}$, then

$$
\begin{aligned}
& \mathcal{Z}_{\alpha}[x] \triangleq\left\{\left(z_{t}\right)_{t \in \overline{0, N}} \in \mathbb{Z} \mid\left(z_{0}=(x, x)\right) \&\left(z_{t} \in \mathbb{M}_{\alpha(t)} \forall t \in \overline{1, N}\right)\right. \\
& \left.\&\left(\operatorname{pr}_{1}\left(z_{s}\right) \in A_{\alpha(s)}\left(\operatorname{pr}_{2}\left(z_{s-1}\right),\{\alpha(l): l \in \overline{s, N}\}\right) \forall s \in \overline{1, N}\right)\right\} .
\end{aligned}
$$

It is easy to verify that (2.8) corresponds to [2, (4)] (for $x=x^{0}$ in $[2,(4)]$ ). By analogy with [2], we give a natural extension of (2.8) to the case when the index set $\overline{1, N}$ is replaced by $K \in \mathfrak{N}$. For $K \in \mathfrak{N}$, we introduce the set $\mathbb{Z}_{K}$ of all tuples

$$
\left(z_{t}\right)_{t \in \overline{0,|K|}}: \overline{0,|K|} \longrightarrow(\mathbb{X} \cup \mathbf{X}) \times \mathbf{X}
$$

Then, for $x \in \mathbf{X}, K \in \mathfrak{N}$, and $\alpha \in(\mathrm{bi})[K]$, we suppose that

$$
\begin{array}{r}
\mathcal{Z}(x, K, \alpha) \triangleq\left\{\left(z_{t}\right)_{t \in \overline{0,|K|}} \in \mathbb{Z}_{K} \mid\left(z_{0}=(x, x)\right) \&\left(z_{t} \in \mathbb{M}_{\alpha(t)} \forall t \in \overline{1,|K|}\right)\right. \\
\left.\&\left(\operatorname{pr}_{1}\left(z_{s}\right) \in A_{\alpha(s)}\left(\operatorname{pr}_{2}\left(z_{s-1}\right),\{\alpha(l): l \in \overline{s,|K|}\}\right) \forall s \in \overline{1,|K|}\right)\right\} ;
\end{array}
$$

see $[2,(5)]$. It is verified by induction that (2.9) is a nonempty set. Besides, relations (2.3) are finite. Therefore,

$$
\mathcal{Z}(x, K, \alpha) \in \operatorname{Fin}\left(\mathbb{Z}_{K}\right) \quad \forall x \in \mathbf{X} \quad \forall K \in \mathfrak{N} \quad \forall \alpha \in(\mathrm{bi})[K] .
$$

Moreover, $\mathcal{Z}_{\alpha}[x]=\mathcal{Z}(x, \overline{1, N}, \alpha) \forall x \in X \forall \alpha \in \mathbb{P}$. As a result, by (2.10),

$$
\mathcal{Z}_{\alpha}[x] \in \operatorname{Fin}(\mathbb{Z}) \quad \forall x \in X^{0} \quad \forall \alpha \in \mathbb{P} .
$$

Now, we introduce a modification of mappings (2.4). Namely, for $j \in \overline{1, N}, x \in \mathbf{X} \backslash \mathbf{M}_{j}$, and $\tilde{K} \in \mathfrak{N}^{(j)}$, we suppose that

$$
\mathbb{A}_{j}(x, \tilde{K}) \triangleq\left\{z \in \mathbb{M}_{j} \mid \operatorname{pr}_{1}(z) \in A_{j}(x, \tilde{K})\right\}
$$

Then, by (2.9) and (2.11), we get that

$$
\begin{gathered}
\mathcal{Z}(x, K, \alpha)=\left\{\left(z_{t}\right)_{t \in \overline{0,|K|}} \in \mathbb{Z}_{K} \mid\left(z_{0}=(x, x)\right)\right. \\
\left.\&\left(z_{t} \in \mathbb{A}_{\alpha(t)}\left(\operatorname{pr}_{2}\left(z_{t-1}\right),\{\alpha(l): l \in \overline{t,|K|}\}\right) \forall t \in \overline{1,|K|}\right)\right\}
\end{gathered}
$$

for $x \in \mathbf{X}, K \in \mathfrak{N}$, and $\alpha \in(\mathrm{bi})[K]$. As a particular case of (2.12), the following representation holds for $x \in X^{0}$ and $\alpha \in \mathbb{P}$ :

$$
\mathcal{Z}_{\alpha}[x]=\left\{\left(z_{t}\right)_{t \in \overline{0, N}} \in \mathbb{Z} \mid\left(z_{0}=(x, x)\right) \&\left(z_{t} \in \mathbb{A}_{\alpha(t)}\left(\operatorname{pr}_{2}\left(z_{t-1}\right),\{\alpha(l): l \in \overline{t, N}\}\right) \forall t \in \overline{1, N}\right)\right\} .
$$


By analogy with $[6,(2.11)]$, for $x \in X^{0}$, we set

$$
\tilde{D}[x] \triangleq\left\{(\alpha, \mathbf{z}) \in \mathbf{A} \times \mathbb{Z} \mid \mathbf{z} \in \mathcal{Z}_{\alpha}[x]\right\} .
$$

Of course, $\tilde{D}[x] \in \operatorname{Fin}(\mathbf{A} \times \mathbb{Z}) \forall x \in X^{0}$. Finally,

$$
\mathbf{D} \triangleq\left\{(\alpha, \mathbf{z}, x) \in \mathbf{A} \times \mathbb{Z} \times X^{0} \mid(\alpha, \mathbf{z}) \in \tilde{D}[x]\right\} \in \operatorname{Fin}\left(\mathbf{A} \times \mathbb{Z} \times X^{0}\right) .
$$

In what follows, we suppose that $\mathbf{M} \triangleq \bigcup_{i=1}^{N} \mathbf{M}_{i}$; of course, $\mathbf{M} \in \operatorname{Fin}(\mathbf{X})$.

Cost functions. We fix the following $N+2$ functions:

$$
\mathbf{c} \in \mathcal{R}_{+}[\mathbf{X} \times \mathbb{X} \times \mathfrak{N}], \quad c_{1} \in \mathcal{R}_{+}\left[\mathbb{M}_{1} \times \mathfrak{N}\right], \quad \ldots, \quad c_{N} \in \mathcal{R}_{+}\left[\mathbb{M}_{1} \times \mathfrak{N}\right], \quad f \in \mathcal{R}_{+}\left[\mathbf{M} \times X^{0}\right]
$$

We use the function $\mathbf{c}$ to estimate the (exterior) permutations between megacities and from $X^{0}$ to megacities. We use the functions $c_{j}, j \in \overline{1, N}$, to estimate the (interior) works connected with visiting $M_{j}$. Finally, $f$ estimates the terminal state of our process (the point $x_{2}^{(N)}$ in $(2.7)$ ). In what follows, we consider only an additive criterion. For $x \in X^{0}, \alpha \in \mathbb{P}$, and $\left(z_{t}\right)_{t \in \overline{0, N}} \in \mathcal{Z}_{\alpha}[x]$, we consider

$$
\begin{gathered}
\mathfrak{C}_{\alpha}\left[\left(z_{t}\right)_{t \in \overline{0, N}} \mid x\right] \triangleq \sum_{t=1}^{N}\left[\mathbf{c}\left(\operatorname{pr}_{2}\left(z_{t-1}\right), \operatorname{pr}_{1}\left(z_{t}\right),\{\alpha(k): k \in \overline{t, N}\}\right)+c_{\alpha(t)}\left(z_{t},\{\alpha(k): k \in \overline{t, N}\}\right)\right] \\
+f\left(\operatorname{pr}_{2}\left(z_{N}\right), x\right) \in \mathbb{R}_{+}
\end{gathered}
$$

as a base for the criterion in an $x$-problem ( $x$ is a starting point). For $x \in X^{0}$, we obtain the following $x$-problem:

$$
\mathfrak{C}_{\alpha}[\mathbf{z} \mid x] \longrightarrow \min , \quad(\alpha, \mathbf{z}) \in \tilde{D}[x]
$$

for this problem, the define the extremum $V[x] \in \mathbb{R}_{+}$as the smallest of the numbers $\mathfrak{C}_{\alpha}[\mathbf{z} \mid x]$, $(\alpha, \mathbf{z}) \in \tilde{D}[x]$. Moreover, for $x \in X^{0}$,

$$
(\mathrm{SOL})[x] \triangleq\left\{(\alpha, \mathbf{z}) \in \tilde{D}[x] \mid \mathfrak{C}_{\alpha}[\mathbf{z} \mid x]=V[x]\right\} \in \mathcal{P}^{\prime}(\tilde{D}[x])
$$

is the set of all optimal solutions of problem (2.17).

Now, we introduce the following complete problem:

$$
\mathfrak{C}_{\alpha}[\mathbf{z} \mid x] \longrightarrow \min , \quad(\alpha, \mathbf{z}, x) \in \mathbf{D} .
$$

For this problem, the global extremum is defined as

$$
\mathbb{V} \triangleq \min _{(\alpha, \mathbf{z}, x) \in \mathbf{D}} \mathfrak{C}_{\alpha}[\mathbf{z} \mid x] \in \mathbb{R}_{+}
$$

and the set of all optimal solutions is

$$
\mathbf{S O L} \triangleq\left\{(\alpha, \mathbf{z}, x) \in \mathbf{D} \mid \mathfrak{C}_{\alpha}[\mathbf{z} \mid x]=\mathbb{V}\right\} \in \operatorname{Fin}(\mathbf{D}) .
$$

The following representation of $\mathbb{V}(2.20)$ is also useful:

$$
\mathbb{V} \triangleq \min _{x \in X^{0}} V[x]
$$

In this paper, we consider only the possibilities of DP as a method for investigating problems (2.17) and (2.19). We keep in mind a variant of DP, which is a development of a scheme from [1]. Using representation (2.21), we consider the problem

$$
V[x] \longrightarrow \min , \quad x \in X^{0} .
$$

This problem allows us to obtain (see (2.21)) important properties of solutions to problem (2.19) in terms of $x$-problems (2.17), $x \in X^{0}$. 


\section{Dynamic programming}

For the DP procedure developing an approach of [1], the dependence of the terminal function $f$ on the starting point is an essentially complicated circumstance. Indeed, its own version of DP is required for every $x \in X^{0}$ (we keep in mind the search of optimal solutions to problem (2.19)). Therefore, we first consider a simpler case when the terminal function is independent of points from $X^{0}$. More precisely, in this section, we fix

$$
\mathbf{f} \in \mathcal{R}_{+}[\mathbf{M}]
$$

Now, we introduce analogs of problems (2.17) and (2.19) corresponding to the change $f \longrightarrow \mathbf{f}$. For this, we replace $(2.16)$ by the following expression for $x \in X^{0}, \alpha \in \mathbb{P}$, and $\left(z_{t}\right)_{t \in \overline{0, N}} \in \mathcal{Z}_{\alpha}[x]$ :

$$
\begin{gathered}
\hat{\mathfrak{C}}_{\alpha}\left[\left(z_{t}\right)_{t \in \overline{0, N}} \mid \mathbf{f}\right] \triangleq \sum_{t=1}^{N}\left[\mathbf{c}\left(\operatorname{pr}_{2}\left(z_{t-1}\right), \operatorname{pr}_{1}\left(z_{t}\right),\{\alpha(k): k \in \overline{t, N}\}\right)+c_{\alpha(t)}\left(z_{t},\{\alpha(k): k \in \overline{t, N}\}\right)\right] \\
+\mathbf{f}\left(\operatorname{pr}_{2}\left(z_{N}\right)\right) \in \mathbb{R}_{+} .
\end{gathered}
$$

Then, (2.17) becomes the following problem. For $x \in X^{0}$, we consider the following (auxiliary) $x$-problem:

$$
\hat{\mathfrak{C}}_{\alpha}[\mathbf{z} \mid \mathbf{f}] \longrightarrow \min , \quad(\alpha, \mathbf{z}) \in \tilde{D}[x]
$$

for problem (3.3), we introduce the extremum $\hat{V}[x \mid \mathbf{f}]$ as the smallest of the numbers $\hat{\mathfrak{C}}_{\alpha}[\mathbf{z} \mid \mathbf{f}]$, $(\alpha, \mathbf{z}) \in \tilde{D}[x]$. Similarly, we replace (2.19) by the following (complete) problem:

$$
\hat{\mathfrak{C}}_{\alpha}[\mathbf{z} \mid \mathbf{f}] \longrightarrow \min , \quad(\alpha, \mathbf{z}, x) \in \mathbf{D} .
$$

For this problem, we consider the extremum

$$
\hat{\mathbb{V}}[\mathbf{f}] \triangleq \min _{(\alpha, \mathbf{z}, x) \in \mathbf{D}} \hat{\mathfrak{C}}_{\alpha}[\mathbf{z} \mid \mathbf{f}] \in \mathbb{R}_{+}
$$

Moreover, by (2.15) and (3.5), we get that

$$
\hat{\mathbb{V}}[\mathbf{f}]=\min _{x \in X^{0}} \hat{V}[x \mid \mathbf{f}] .
$$

Now, for $x \in X^{0}$, we introduce the set

$$
\hat{\mathbb{S}}[x \mid \mathbf{f}] \triangleq\left\{(\alpha, \mathbf{z}) \in \tilde{D}[x] \mid \hat{\mathfrak{C}}_{\alpha}[\mathbf{z} \mid \mathbf{f}]=\hat{V}[x \mid \mathbf{f}]\right\} \in \operatorname{Fin}(\tilde{D}[x])
$$

of all optimal solutions to problem (3.3). Finally,

$$
\hat{\mathbf{S}}[\mathbf{f}] \triangleq\left\{(\alpha, \mathbf{z}, x) \in \mathbf{D} \mid \hat{\mathfrak{C}}_{\alpha}[\mathbf{z} \mid \mathbf{f}]=\hat{\mathbb{V}}[\mathbf{f}]\right\} \in \operatorname{Fin}(\mathbf{D})
$$

is the set of all optimal solutions to problem (3.4).

Returning to (2.12), we introduce partial routes admissible by deletion (tasks from a list). Namely, we introduce a mapping $\mathbf{I}$ operating in $\mathfrak{N}$ by the following rule for $K \in \mathfrak{N}$ :

$$
\mathbf{I}(K) \triangleq K \backslash\left\{\operatorname{pr}_{2}(z): z \in \Xi[K]\right\},
$$

where $\Xi[K] \triangleq\left\{z \in \mathbf{K} \mid\left(\operatorname{pr}_{1}(z) \in K\right) \&\left(\operatorname{pr}_{2}(z) \in K\right)\right\}$. Suppose that, for $\mathbb{K} \in \mathfrak{N}$,

$$
(\mathbf{I}-\mathrm{bi})[\mathbb{K}] \triangleq\{\alpha \in(\mathrm{bi})[\mathbb{K}] \mid \alpha(s) \in \mathbf{I}(\{\alpha(t): t \in \overline{s,|\mathbb{K}|}\}) \quad \forall s \in \overline{1,|\mathbb{K}|}\} .
$$


Then, by statements of $[3$, Part 2], $(\mathbf{I}-$ bi) $[K] \neq \varnothing$ for $K \in \mathfrak{N}$; moreover,

$$
\begin{gathered}
\mathbf{A}=(\mathbf{I}-\mathrm{bi})[\overline{1, N}]=\{\alpha \in \mathbb{P} \mid(\alpha(1) \in \mathbf{I}(\overline{1, N})) \\
\&(\alpha(k) \in \mathbf{I}(\overline{1, N} \backslash\{\alpha(l): l \in \overline{1, k-1}\}) \forall k \in \overline{2, N})\} .
\end{gathered}
$$

Thus, according to (3.1), the admissibility by precedence and the admissibility by deletion are identical for complete routes. Now, for $x \in \mathbf{X}$ and $K \in \mathfrak{N}$, we consider the corresponding partial routing problem. First, we introduce a partial criterion. If $x \in \mathbf{X}, K \in \mathfrak{N}, \alpha \in(\mathrm{bi})[K]$, and $\left(z_{t}\right)_{t \in \overline{0,|K|}} \in \mathcal{Z}(x, K, \alpha)$, then

$$
\begin{gathered}
\hat{\mathfrak{C}}_{\alpha}^{*}\left[\left(z_{t}\right)_{t \in \overline{0,|K|} \mid} \mathbf{f} ; K\right] \triangleq \sum_{s=1}^{|K|}\left[\mathbf{c}\left(\operatorname{pr}_{2}\left(z_{s-1}\right), \operatorname{pr}_{1}\left(z_{s}\right),\{\alpha(t): t \in \overline{s,|K|}\}\right)\right. \\
\left.+c_{\alpha(s)}\left(z_{s},\{\alpha(t): t \in \overline{s,|K|}\}\right)\right]+\mathbf{f}\left(\operatorname{pr}_{2}\left(z_{|K|}\right)\right) .
\end{gathered}
$$

Of course, the case $K=\overline{1, N}$ is possible; so, for $\alpha \in \mathbb{P}$ and $\left(z_{t}\right)_{t \in \overline{0, N}} \in \mathcal{Z}_{\alpha}[x]$, the number $\hat{\mathfrak{C}}_{\alpha}^{*}\left[\left(z_{t}\right)_{t \in \overline{0, N}} \mid \mathbf{f} ; \overline{1, N}\right] \in \mathbb{R}_{+}$is defined. In addition, by (3.2) and (3.9), for $x \in \mathbf{X}, K \in \mathfrak{N}, \alpha \in \mathbb{P}$, and $\mathbf{z} \in \mathcal{Z}_{\alpha}[x]$, we have

$$
\hat{\mathfrak{C}}_{\alpha}[\mathbf{z} \mid \mathbf{f}]=\hat{\mathfrak{C}}_{\alpha}^{*}[\mathbf{z} \mid \mathbf{f} ; \overline{1, N}]
$$

By analogy with (2.13), for $x \in \mathbf{X}$ and $K \in \mathfrak{N}$, we set

$$
\hat{D}^{*}(x, K) \triangleq\left\{(\alpha, \mathbf{z}) \in(\mathbf{I}-\mathrm{bi})[K] \times \mathbb{Z}_{K} \mid \mathbf{z} \in \mathcal{Z}(x, K, \alpha)\right\} \in \operatorname{Fin}\left((\mathbf{I}-\text { bi })[K] \times \mathbb{Z}_{K}\right) .
$$

For $x \in \mathbf{X}$ and $K \in \mathfrak{N}$, consider the following problem:

$$
\hat{\mathfrak{C}}_{\alpha}^{*}[\mathbf{z} \mid \mathbf{f} ; K] \longrightarrow \min , \quad(\alpha, \mathbf{z}) \in \hat{D}^{*}(x, K) ;
$$

$v_{\mathbf{f}}(x, K)$ denotes the smallest of the numbers $\hat{\mathfrak{C}}_{\alpha}^{*}[\mathbf{z} \mid \mathbf{f} ; K],(\alpha, \mathbf{z}) \in \hat{D}^{*}(x, K)$. Note that, by (2.13) and (3.8), we have

$$
\tilde{D}[x]=\hat{D}^{*}(x, \overline{1, N}) \quad \forall x \in X^{0} .
$$

From (3.10) and (3.11), we get that

$$
\hat{V}[x \mid \mathbf{f}]=v_{\mathbf{f}}(x, \overline{1, N}) \quad \forall x \in X^{0} .
$$

Finally, we set

$$
v_{\mathbf{f}}(x, \varnothing) \triangleq \mathbf{f}(x) \quad \forall x \in \mathbf{M} .
$$

Now we construct a function defined on $(\mathbf{X} \times \mathfrak{N}) \cup(\mathbf{M} \times\{\varnothing\})$. Namely,

$$
v_{\mathbf{f}} \in \mathcal{R}_{+}[(\mathbf{X} \times \mathfrak{N}) \cup(\mathbf{M} \times\{\varnothing\})]
$$

is defined by the following conditions:

$$
\left(v_{\mathbf{f}}(x, K) \triangleq \min _{(\alpha, \mathbf{z}) \in \hat{D}^{*}(x, K)} \hat{\mathfrak{C}}_{\alpha}^{*}[\mathbf{z} \mid \mathbf{f} ; K] \quad \forall(x, K) \in \mathbf{X} \times \mathfrak{N}\right) \&\left(v_{\mathbf{f}}(x, \varnothing) \triangleq \mathbf{f}(x) \quad \forall x \in \mathbf{M}\right)
$$

(we use the obvious equality $\mathcal{P}(\overline{1, N})=\mathfrak{N} \cup\{\varnothing\}$ ). By (3.12), we define the value function

$$
\hat{V}[\cdot \mid \mathbf{f}] \triangleq(\hat{V}[x \mid \mathbf{f}])_{x \in X^{0}} \in \mathcal{R}_{+}\left[X^{0}\right] .
$$

Theorem 1. If $x \in \mathbf{X}$ and $K \in \mathfrak{N}$, then

$$
v_{\mathbf{f}}(x, K)=\min _{j \in \mathbf{I}(K)} \min _{z \in \mathbb{A}_{j}(x, K)}\left[\mathbf{c}\left(x, \operatorname{pr}_{1}(z), K\right)+c_{j}(z, K)+v_{\mathbf{f}}\left(\operatorname{pr}_{2}(z), K \backslash\{j\}\right)\right] .
$$


Theorem 1 is extracted from [2, Theorem 1]. Now, we discuss only some peculiarities (note that $[2$, Theorem 1] was proved by analogy with [4, Theorem 5.1]). In [2, Theorem 1], the case of fixed starting point was considered. However, the Bellman function from [2, Sect. 3] can be defined on the set $\mathbf{X} \times \mathcal{P}(\overline{1, N})$ (note that, in [4], not the whole Bellman function was used): we follow a scheme of $\left[2\right.$, Sect. 3] for every starting point from $X^{0}$. In addition, the definition of the Bellman function from [2, Sect. 3] corresponds to (3.14). Thus, Theorem 1 from [2] is true in our case (also note [13, Theorem 1] where this question was also considered). We obtain (3.16). As a particular case, we note the corresponding analog of $[2,(18)]$ : if $x \in X^{0}$, then, by (3.12) and (3.16),

$$
\hat{V}[x \mid \mathbf{f}]=\min _{j \in \mathbf{I}(\overline{1, N})} \min _{z \in \mathbb{A}_{j}(x, \overline{1, N})}\left[\mathbf{c}\left(x, \operatorname{pr}_{1}(z), \overline{1, N}\right)+c_{j}(z, \overline{1, N})+v_{\mathbf{f}}\left(\operatorname{pr}_{2}(z), \overline{1, N} \backslash\{j\}\right)\right] .
$$

As in $[2-4,6]$, to reduce computational complexity, we will only constructbuild special layers of our Bellman function. First, we introduce special subsets of $\overline{1, N}$. We consider these subsets as substantial task lists. Let

$$
\mathcal{G} \triangleq\left\{K \in \mathfrak{N} \mid \forall z \in \mathbf{K} \quad\left(\operatorname{pr}_{1}(z) \in K\right) \Longrightarrow\left(\operatorname{pr}_{2}(z) \in K\right)\right\} .
$$

Let also $\mathcal{G}_{s} \triangleq\{K \in \mathcal{G}|s=| K \mid\} \forall s \in \overline{1, N}$. Then $\left\{\mathcal{G}_{1} ; \ldots ; \mathcal{G}_{N}\right\}$ is a partition of $\mathcal{G} ; \mathcal{G}_{N}=\{\overline{1, N}\}$ and $\mathcal{G}_{1}=\left\{\{t\}: t \in \overline{1, N} \backslash \mathbf{K}_{1}\right\}$, where $\mathbf{K}_{1} \triangleq\left\{\operatorname{pr}_{1}(z): z \in \mathbf{K}\right\}$. Finally (see [2-4,6]),

$$
\mathcal{G}_{s-1}=\left\{K \backslash\{t\}: K \in \mathcal{G}_{s}, t \in \mathbf{I}(K)\right\} .
$$

So, we can implement the procedure $\mathcal{G}_{N} \longrightarrow \mathcal{G}_{N-1} \longrightarrow \cdots \longrightarrow \mathcal{G}_{1}$ (we use (3.18)). Further, we construct sets $\mathcal{D}_{0}, \mathcal{D}_{1}, \ldots, \mathcal{D}_{N}$. Let $\mathcal{D}_{N} \triangleq\left\{(x, \overline{1, N}): x \in X^{0}\right\}$ and

$$
\mathcal{D}_{0} \triangleq\left\{(x, \varnothing): x \in \underset{i \in \overline{1, N} \backslash \mathbf{K}_{1}}{\bigcup_{i}} \mathbf{M}_{i}\right.
$$

For $s \in \overline{1, N-1}$ and $K \in \mathcal{G}_{s}$, we successively construct

$$
\mathcal{J}_{s}(K) \triangleq\left\{j \in \overline{1, N} \backslash K \mid\{j\} \cup K \in \mathcal{G}_{s+1}\right\}, \quad \mathcal{M}_{s}[K] \triangleq \bigcup_{j \in \mathcal{J}_{s}(K)} \mathbf{M}_{j}, \mathbb{D}_{s}[K] \triangleq\left\{(x, K): x \in \mathcal{M}_{s}[K]\right\}
$$

(all these sets are nonempty; see [3, Sect. 4.9]). Finally, for $s \in \overline{1, N-1}$, we set

$$
\mathcal{D}_{s} \triangleq \bigcup_{K \in \mathcal{G}_{s}} \mathbb{D}_{s}[K]
$$

So, all layers $\mathcal{D}_{0}, \mathcal{D}_{1}, \ldots, \mathcal{D}_{N}$ are constructed. We recall that (see $[2,(3.6)]$ )

$$
\left(\operatorname{pr}_{2}(z), K \backslash\{j\}\right) \in \mathcal{D}_{s-1} \quad \forall s \in \overline{1, N} \quad \forall(x, K) \in \mathcal{D}_{s} \quad \forall j \in \mathbf{I}(K) \quad \forall z \in \mathbb{M}_{j} .
$$

Now, we construct the Bellman function layers using Theorem 1 and (3.20). More precisely, we keep in mind the functions

$$
v_{\mathbf{f}}^{(0)} \in \mathcal{R}_{+}\left[\mathcal{D}_{0}\right], \quad v_{\mathbf{f}}^{(1)} \in \mathcal{R}_{+}\left[\mathcal{D}_{1}\right], \quad \ldots, \quad v_{\mathbf{f}}^{(N)} \in \mathcal{R}_{+}\left[\mathcal{D}_{N}\right] .
$$

Using (3.13) and (3.19), we set

$$
v_{\mathbf{f}}^{(0)}(x, \varnothing) \triangleq \mathbf{f}(x) \forall x \in \underset{i \in \overline{1, N} \backslash \mathbf{K}_{1}}{\bigcup} \mathbf{M}_{i}
$$


In general, we define $v_{\mathbf{f}}^{(s)}$ for $s \in \overline{0, N}$ by the following rule:

$$
v_{\mathbf{f}}^{(s)}(x, K) \triangleq v_{\mathbf{f}}(x, K) \quad \forall(x, K) \in \mathcal{D}_{s}
$$

((3.22) is a particular case of (3.23)). Of course, (3.23) is a mathematical definition. Now, we introduce a recurrence procedure for immediate construction of all functions (3.21). Namely, $v_{\mathbf{f}}^{(0)}$ is known (see (3.22)). For $s \in \overline{1, N}$, the transformation $v_{\mathbf{f}}^{(s-1)} \longrightarrow v_{\mathbf{f}}^{(s)}$ is implemented by the rule

$$
v_{\mathbf{f}}^{(s)}(x, K)=\min _{j \in \mathbf{I}(K)} \min _{z \in \mathbb{A}_{j}(x, K)}\left[\mathbf{c}\left(x, \operatorname{pr}_{1}(z), K\right)+c_{j}(z, K)+v_{\mathbf{f}}^{(s-1)}\left(\operatorname{pr}_{2}(z), K \backslash\{j\}\right)\right] \quad \forall(x, K) \in D_{s} .
$$

So, (3.24) defines the following recurrence procedure:

$$
v_{\mathbf{f}}^{(0)} \longrightarrow v_{\mathbf{f}}^{(1)} \longrightarrow \cdots \longrightarrow v_{\mathbf{f}}^{(N)} .
$$

Note that, by $(3.23), v_{\mathbf{f}}^{(N)}(x, K)=v_{\mathbf{f}}(x, K)$ for $(x, K) \in \mathcal{D}_{N}$. Using (3.12) and the representation of $\mathcal{D}_{N}$, we get that

$$
\hat{V}[x \mid \mathbf{f}]=v_{\mathbf{f}}(x, \overline{1, N})=v_{\mathbf{f}}^{(N)}(x, \overline{1, N}) \quad \forall x \in X^{0} .
$$

Here, we note an obvious corollary of (3.17). Namely, by (3.20), we get that, $\left(\operatorname{pr}_{2}(z), \overline{1, N} \backslash\{j\}\right) \in \mathcal{D}_{N-1}$ for $x \in X^{0}, j \in \mathbf{I}(\overline{1, N})$, and $z \in \mathbb{M}_{j}$. Therefore, by (3.17), we have

$$
\hat{V}[x \mid \mathbf{f}]=\min _{j \in \mathbf{I}(\overline{1, N})} \min _{z \in \mathbb{A}_{j}(x, \overline{1, N})}\left[\mathbf{c}\left(x, \operatorname{pr}_{1}(z), \overline{1, N}\right)+c_{j}(z, \overline{1, N})+v_{\mathbf{f}}^{(N-1)}\left(\operatorname{pr}_{2}(z), \overline{1, N} \backslash\{j\}\right)\right] \quad \forall x \in X^{0}
$$

(we use (2.11) and (3.23)). So, we can construct $\hat{V}[\cdot \mid \mathbf{f}](3.15)$. As a corollary, we can find $\hat{\mathbb{V}}[\mathbf{f}](3.8)$ and a point $x^{0} \in X^{0}$ for which $\hat{V}\left[x^{0} \mid \mathbf{f}\right]=\hat{\mathbb{V}}[\mathbf{f}]$. So, $x^{0}$ is an optimal starting point in the problem with the terminal function $\mathbf{f}$.

Now, we will build an optimal solution to problem (3.7). We fix a point $x^{0}$ with this optimality property. Let $\mathbf{z}^{(0)} \triangleq\left(x^{0}, x^{0}\right)$. Using (3.24), we choose $\eta_{1} \in \mathbf{I}(\overline{1, N})$ and $\mathbf{z}^{(1)} \in \mathbb{A}_{\eta_{1}}\left(x^{0}, \overline{1, N}\right)$ for which

$$
\hat{V}\left[x^{0} \mid \mathbf{f}\right]=\mathbf{c}\left(x^{0}, \operatorname{pr}_{1}\left(\mathbf{z}^{(1)}, \overline{1, N}\right)+c_{\eta_{1}}\left(\mathbf{z}^{(1)}, \overline{1, N}\right)+v_{\mathbf{f}}^{(N-1)}\left(\operatorname{pr}_{2}\left(\mathbf{z}^{(1)}\right), \overline{1, N} \backslash\left\{\eta_{1}\right\}\right)\right.
$$

(we follow a procedure of $\left[2\right.$, Sect. 4]). Then, $\left(\operatorname{pr}_{2}\left(\mathbf{z}^{(1)}\right), \overline{1, N} \backslash\left\{\eta_{1}\right\}\right) \in \mathcal{D}_{N-1}$, and therefore (see $(3.24))$

$$
\begin{gathered}
v_{\mathbf{f}}^{(N-1)}\left(\operatorname{pr}_{2}\left(\mathbf{z}^{(1)}\right), \overline{1, N} \backslash\left\{\eta_{1}\right\}\right)=\min _{j \in \mathbf{I}\left(\overline{1, N} \backslash\left\{\eta_{1}\right\}\right)} \min _{z \in \mathbb{A}_{j}\left(\operatorname{pr}_{2}\left(\mathbf{z}^{(1)}, \overline{1, N} \backslash\left\{\eta_{1}\right\}\right)\right.}\left[\mathbf{c}\left(\operatorname{pr}_{2}\left(\mathbf{z}^{(1)}\right), \operatorname{pr}_{1}(z), \overline{1, N} \backslash\left\{\eta_{1}\right\}\right)\right. \\
\left.+c_{j}\left(z, \overline{1, N} \backslash\left\{\eta_{1}\right\}\right)+v_{\mathbf{f}}^{(N-2)}\left(\operatorname{pr}_{2}(z), \overline{1, N} \backslash\left\{\eta_{1} ; j\right\}\right)\right]
\end{gathered}
$$

of course, we take into account that, by (3.20),

$$
\left(\operatorname{pr}_{2}(z), \overline{1, N} \backslash\left\{\eta_{1} ; j\right\}\right)=\left(\operatorname{pr}_{2}(z),\left(\overline{1, N} \backslash\left\{\eta_{1}\right\}\right) \backslash\{j\}\right) \in \mathcal{D}_{N-2} \quad \forall j \in \mathbf{I}\left(\overline{1, N} \backslash\left\{\eta_{1}\right\}\right) \quad \forall z \in \mathbb{M}_{j} .
$$

Now, using (3.27), we choose $\eta_{2} \in \mathbf{I}\left(\overline{1, N} \backslash\left\{\eta_{1}\right\}\right)$ and $\mathbf{z}^{(2)} \in \mathbb{A}_{\eta_{2}}\left(\operatorname{pr}_{2}\left(\mathbf{z}^{(1)}, \overline{1, N} \backslash\left\{\eta_{1}\right\}\right)\right.$ for which

$$
\begin{gathered}
v_{\mathbf{f}}^{(N-1)}\left(\operatorname{pr}_{2}\left(\mathbf{z}^{(1)}\right), \overline{1, N} \backslash\left\{\eta_{1}\right\}\right)=\mathbf{c}\left(\operatorname{pr}_{2}\left(\mathbf{z}^{(1)}\right), \operatorname{pr}_{1}\left(\mathbf{z}^{(2)}\right), \overline{1, N} \backslash\left\{\eta_{1}\right\}\right)+c_{\eta_{2}}\left(\mathbf{z}^{(2)}, \overline{1, N} \backslash\left\{\eta_{1}\right\}\right) \\
+v_{\mathbf{f}}^{(N-2)}\left(\operatorname{pr}_{2}\left(\mathbf{z}^{(2)}\right), \overline{1, N} \backslash\left\{\eta_{1} ; \eta_{2}\right\}\right) .
\end{gathered}
$$


From (3.26) and (3.28), we get that

$$
\begin{gathered}
\hat{V}\left[x^{0} \mid \mathbf{f}\right]=\mathbf{c}\left(\operatorname{pr}_{2}\left(\mathbf{z}^{(0)}\right), \operatorname{pr}_{1}\left(\mathbf{z}^{(1)}\right), \overline{1, N}\right)+\mathbf{c}\left(\operatorname{pr}_{2}\left(\mathbf{z}^{(1)}\right), \operatorname{pr}_{1}\left(\mathbf{z}^{(2)}\right), \overline{1, N} \backslash\left\{\eta_{1}\right\}\right)+c_{\eta_{1}}\left(\mathbf{z}^{(1)}, \overline{1, N}\right) \\
+c_{\eta_{2}}\left(\mathbf{z}^{(2)}, \overline{1, N} \backslash\left\{\eta_{1}\right\}\right)+v_{\mathbf{f}}^{(N-2)}\left(\operatorname{pr}_{2}\left(\mathbf{z}^{(2)}\right), \overline{1, N} \backslash\left\{\eta_{1} ; \eta_{2}\right\}\right) .
\end{gathered}
$$

Of course, $\eta_{1} \neq \eta_{2}$. Further, procedures similar to (3.26) and (3.28) must be continued until $\overline{1, N}$ is exhausted. As a result (see [2, Sect. 4]), $\eta \triangleq\left(\eta_{j}\right)_{j \in \overline{1, N}} \in \mathbf{A}$ and $\left(\mathbf{z}^{(j)}\right)_{j \in \overline{0, N}} \in \mathcal{Z}_{\eta}\left[x^{0}\right]$ with the property

$$
\hat{\mathfrak{C}}_{\eta}\left[\left(\mathbf{z}^{(j)}\right)_{j \in \overline{0, N}} \mid \mathbf{f}\right]=\hat{V}\left[x^{0} \mid \mathbf{f}\right]
$$

will be build. Using the optimality of $x^{0}$, we get from (3.29) that

$$
\left(\eta,\left(\mathbf{z}^{(j)}\right)_{j \in \overline{0, N}}, x^{0}\right) \in \hat{\mathbf{S}}[\mathbf{f}]
$$

(indeed, by (3.6) and (3.29), $\left.\left(\eta,\left(\mathbf{z}^{(j)}\right)_{j \in \overline{0, N}}\right) \in \hat{\mathbb{S}}\left[x^{0} \mid \mathbf{f}\right]\right)$. So, for problem (3.4), we found the global extremum $\hat{\mathbb{V}}[\mathbf{f}]$ and an optimal solution. In what follows, we consider (3.4) as an auxiliary problem.

\section{Individual dynamic programming}

Now, we return to problem (2.19) for which the terminal component of our criterion corresponds to (2.16). In this case, the DP procedure is "attached" to the starting point. More precisely, its own DP procedure is required for every point $x \in X^{0}$. This procedure corresponds to $[4,5]$. Therefore, we consider it very briefly. We use $\mathcal{G}_{1}, \mathcal{G}_{2}, \ldots, \mathcal{G}_{N}$ and layers $\mathcal{D}_{0}, \mathcal{D}_{1}, \ldots, \mathcal{D}_{N-1}$ from Section 3. For $x \in X^{0}$, we fix $D_{N}(x) \triangleq\{(x, \overline{1, N})\}$ and obtain a singleton attached to the starting point $x$. To universal notation, we set

$$
\left(D_{j}(x) \triangleq \mathcal{D}_{j} \forall j \in \overline{0, N-1}\right) \&\left(D_{N}(x) \triangleq\{(x, \overline{1, N})\}\right),
$$

where $x \in X^{0}$. By (4.1), we have only one singular layer (corresponding to the index $N$ ). Recall (see [5]) that, for $x \in X^{0}$,

$$
\left(\operatorname{pr}_{2}(z), K \backslash\{j\}\right) \in D_{s-1}(x) \quad \forall s \in \overline{1, N} \quad \forall(y, K) \in D_{s}(x) \quad \forall j \in \mathbf{I}(K) \quad \forall z \in \mathbb{M}_{j} .
$$

So, in (4.2), we have a natural analog of (3.20).

Note that, to solve the $x$-problem for $x \in X^{0}$, we can use a DP procedure from [2] (a DP procedure from [5] is a particular case of that from [2]). Now, we will restrict ourselves to the algorithmic version of the presentation.

For more concise notation, we fix $x \in X^{0}$ unless otherwise stated. Recall that $D_{j}(x) \neq \varnothing$ $\forall j \in \overline{0, N}$. Now, we introduce a recurrence procedure for constructing layers of the Bellman function

$$
\mathbf{v}_{0}[x] \in \mathcal{R}_{+}\left[D_{0}(x)\right], \quad \mathbf{v}_{1}[x] \in \mathcal{R}_{+}\left[D_{1}(x)\right], \quad \ldots, \quad \mathbf{v}_{N}[x] \in \mathcal{R}_{+}\left[D_{N}(x)\right] .
$$

Let

$$
\mathbf{v}_{0}[x](y, \varnothing) \triangleq f(y, x) \forall y \in \bigcup_{j \in \overline{1, N} \backslash \mathbf{K}_{1}} \mathbf{M}_{j} ;
$$

we use the obvious equality $D_{0}(x)=\mathcal{D}_{0}$, see (3.19). Further, for $s \in \overline{1, N}$, transformation of $\mathbf{v}_{s-1}[x] \in \mathcal{R}_{+}\left[D_{s-1}(x)\right]$ to $\mathbf{v}_{s}[x] \in \mathcal{R}_{+}\left[D_{s}(x)\right]$ is defined by the following rule for $(y, K) \in D_{s}(x)$ :

$$
\mathbf{v}_{s}[x](y, K) \triangleq \min _{j \in \mathbf{I}(K)} \min _{z \in \mathbb{A}_{j}(y, K)}\left[\mathbf{c}\left(y, \operatorname{pr}_{1}(z), K\right)+c_{j}(z, K)+\mathbf{v}_{s-1}[x]\left(\operatorname{pr}_{2}(z), K \backslash\{j\}\right)\right]
$$


(we use (4.2)). In this section, we suppose that functions (4.3) are implemented by the procedure

$$
\mathbf{v}_{0}[x] \longrightarrow \mathbf{v}_{1}[x] \longrightarrow \cdots \longrightarrow \mathbf{v}_{N}[x]
$$

More precisely, we have $\mathbf{v}_{0}[x]$ (see (4.4)) and construct $\mathbf{v}_{1}[x]$ using (4.5) for $s=1$ and so on. Here, there exists a unique Bellman function (see [2, Sect. 3]) for which functions (4.3) are implemented as a contraction system; this property is similar to (3.23). But now we restrict ourselves to the recurrence procedure (4.6).

The function $\mathbf{v}_{N}[x]$ is defined by the unique value

$$
\mathbf{v}_{N}[x](x, \overline{1, N})=V[x]
$$

similar to (3.25); in this connection, see $[2,(2.3)]$. We obtain an extremum of the $x$-problem (2.17). Now we will very briefly consider the procedure that implements an element of (SOL) $x](2.18)$.

Suppose that $\mathbf{h}^{(0)} \triangleq(x, x)$. Using an analog of $[2,(23)]$, we choose $\zeta_{1} \in \mathbf{I}(\overline{1, N})$ and $\mathbf{h}^{(1)} \in \mathbb{A}_{\zeta_{1}}(x, \overline{1, N})$ for which

$$
V[x]=\mathbf{c}\left(x, \operatorname{pr}_{1}\left(\mathbf{h}^{(1)}\right), \overline{1, N}\right)+c_{\zeta_{1}}\left(\mathbf{h}^{(1)}, \overline{1, N}\right)+\mathbf{v}_{N-1}[x]\left(\operatorname{pr}_{2}\left(\mathbf{h}^{(1)}\right), \overline{1, N} \backslash\left\{\zeta_{1}\right\}\right) ;
$$

by (4.2), we have $\left(\operatorname{pr}_{2}\left(\mathbf{h}^{(1)}\right), \overline{1, N} \backslash\left\{\zeta_{1}\right\}\right) \in D_{N-1}(x)$. From (4.5), we get that

$$
\begin{gathered}
\mathbf{v}_{N-1}[x]\left(\operatorname{pr}_{2}\left(\mathbf{h}^{(1)}\right), \overline{1, N} \backslash\left\{\zeta_{1}\right\}\right)=\min _{j \in \mathbf{I}\left(\overline{1, N} \backslash\left\{\zeta_{1}\right\}\right)} \min _{z \in \mathbb{A}_{j}\left(\operatorname{pr}_{2}\left(\mathbf{h}^{(1)}\right), \overline{1, N} \backslash\left\{\zeta_{1}\right\}\right)}\left[\mathbf { c } \left(\operatorname{pr}_{2}\left(\mathbf{h}^{(1)}\right), \operatorname{pr}_{1}(z),\right.\right. \\
\left.\left.\overline{1, N} \backslash\left\{\zeta_{1}\right\}\right)+c_{j}\left(z, \overline{1, N} \backslash\left\{\zeta_{1}\right\}\right)+\mathbf{v}_{N-2}[x]\left(\operatorname{pr}_{2}(z), \overline{1, N} \backslash\left\{\zeta_{1} ; j\right\}\right)\right] .
\end{gathered}
$$

Using (4.9), we choose $\zeta_{2} \in \mathbf{I}\left(\overline{1, N} \backslash\left\{\zeta_{1}\right\}\right)$ and $\mathbf{h}^{(2)} \in \mathbb{A}_{\zeta_{2}}\left(\operatorname{pr}_{2}\left(\mathbf{h}^{(1)}\right), \overline{1, N} \backslash\left\{\zeta_{1}\right\}\right)$ for which

$$
\begin{gathered}
\mathbf{v}_{N-1}[x]\left(\operatorname{pr}_{2}\left(\mathbf{h}^{(1)}\right), \overline{1, N} \backslash\left\{\zeta_{1}\right\}\right)=\mathbf{c}\left(\operatorname{pr}_{2}\left(\mathbf{h}^{(1)}\right), \operatorname{pr}_{1}\left(\mathbf{h}^{(2)}\right), \overline{1, N} \backslash\left\{\zeta_{1}\right\}\right) \\
+c_{\zeta_{2}}\left(\mathbf{h}^{(2)}, \overline{1, N} \backslash\left\{\zeta_{1}\right\}\right)+\mathbf{v}_{N-2}[x]\left(\operatorname{pr}_{2}\left(\mathbf{h}^{(2)}\right), \overline{1, N} \backslash\left\{\zeta_{1} ; \zeta_{2}\right\}\right) .
\end{gathered}
$$

By (4.2), we have $\left(\operatorname{pr}_{2}\left(\mathbf{h}^{(2)}\right), \overline{1, N} \backslash\left\{\zeta_{1} ; \zeta_{2}\right\}\right)=\left(\operatorname{pr}_{2}\left(\mathbf{h}^{(2)}\right),\left(\overline{1, N} \backslash\left\{\zeta_{1}\right\}\right) \backslash\left\{\zeta_{2}\right\}\right) \in D_{N-2}(x)$. From (4.8) and (4.10), we obtain the following equality:

$$
\begin{gathered}
V[x]=\mathbf{c}\left(x, \operatorname{pr}_{1}\left(\mathbf{h}^{(1)}\right), \overline{1, N}\right)+\mathbf{c}\left(\operatorname{pr}_{2}\left(\mathbf{h}^{(1)}\right), \operatorname{pr}_{1}\left(\mathbf{h}^{(2)}\right), \overline{1, N} \backslash\left\{\zeta_{1}\right\}\right)+c_{\zeta_{1}}\left(\mathbf{h}^{(1)}, \overline{1, N}\right) \\
+c_{\zeta_{2}}\left(\mathbf{h}^{(2)}, \overline{1, N} \backslash\left\{\zeta_{1}\right\}\right)+\mathbf{v}_{N-2}[x]\left(\operatorname{pr}_{2}\left(\mathbf{h}^{(2)}\right), \overline{1, N} \backslash\left\{\zeta_{1} ; \zeta_{2}\right\}\right) .
\end{gathered}
$$

Further, procedures similar to (4.8) and (4.10) must be continued until $\overline{1, N}$ is exhausted. As a result, we get (see [2, Sect. 4]) that

$$
\zeta \triangleq\left(\zeta_{j}\right)_{j \in \overline{1, N}} \in \mathbf{A}:\left(\mathbf{h}^{(j)}\right)_{j \in \overline{0, N}} \in \mathcal{Z}_{\zeta}[x]
$$

in addition, $\mathfrak{C}_{\zeta}\left[\left(\mathbf{h}^{(j)}\right)_{j \in \overline{0, N}} \mid x\right]=V[x]$ (the latter equality for $N=2$ follows from (4.11)). Thus, $\left(\zeta,\left(\mathbf{h}^{(j)}\right)_{j \in \overline{0, N}}\right) \in(\mathrm{SOL})[x]$.

Remark 1. Now, we return to procedure (4.6). If our goal is only to define $V[x]$ (4.7), then we can use the following analog of a procedure from [15]. Namely, we consider the issue of some memory savings. We have $\mathbf{v}_{0}[x]$. Let $s \in \overline{1, N}$ and $\mathbf{v}_{s-1}[x]$ be known. Then, by (4.5), we construct $\mathbf{v}_{s}[x]$. If $s=N$, then our procedure is complete. If $s<N$, then $\mathbf{v}_{s-1}[x]$ is annihilated and replaced by $\mathbf{v}_{s}[x]$. So, in the computer memory for this scheme there situated only one layer of the Bellman function. As a result, we obtain (4.7). This procedure must be implemented for every $x \in X^{0}$. In addition, we can determine all values $V[x], x \in X^{0}$ without using (4.8)-(4.11). As a result, we can find $\mathbb{V}(2.21)$ and $x^{0} \in X^{0}$ for which $V\left[x^{0}\right]=\mathbb{V}$. 
Further, we implement scheme (4.8)-(4.11) for $x=x^{0}$. Since $\left(\zeta,\left(\mathbf{h}^{(j)}\right)_{j \in \overline{0, N}}, x^{0}\right)$ (see (4.12)), we obtain an optimal solution to problem (2.19). Our scheme includes solutions to all $x$-problems (2.17), although using (4.8)-(4.11) is required once. We get a laborious procedure. Therefore, below we will consider an approach in which it is assumed that the enumeration of not all points $x \in X^{0}$ is realized. Namely, we introduce special majorizing and minorazing problems for which simpler versions of DP procedures can be used.

\section{Auxiliary routing problems and enumeration problem}

First, we introduce a special variant of the problem from Section 3. Consider a function $\mathbf{O} \in \mathcal{R}_{+}[\mathbf{M}]$ such that

$$
\mathbf{O}(x) \triangleq 0 \quad \forall x \in \mathbf{M} .
$$

Now, we implement the constructions from Section 3 for

$$
\mathbf{f}=\mathbf{O} \text {. }
$$

But, in this implementation, we first restrict ourselves to the construction of the function

$$
\hat{V}[\cdot \mid \mathbf{O}]=(\hat{V}[x \mid \mathbf{O}])_{x \in X^{0}} \in \mathcal{R}_{+}\left[X^{0}\right] .
$$

For this, we use a variant of DP similar to that in Remark 1. More precisely, we use the layers $\mathcal{D}_{0}, \mathcal{D}_{1}, \ldots, \mathcal{D}_{N}$ from Section 3 with property $(3.20)$.

Further, we construct functions (3.21) for (5.2). Namely, $\left.\left.v_{\mathbf{O}}^{(0)} \in \mathcal{R}_{+}\right] \mathcal{D}_{0}\right]$ is defined by the rule $v_{\mathbf{O}}^{(0)}(x, K)=0 \forall(x, K) \in \mathcal{D}_{0}$; thus, $v_{\mathbf{O}}^{(0)}$ is identically equal to zero. If $s \in \overline{1, N}$ and $v_{\mathbf{O}}^{(s-1)} \in \mathcal{R}_{+}\left[\mathcal{D}_{s-1}\right]$ is known, then we define $v_{\mathbf{O}}^{(s)} \in \mathcal{R}_{+}\left[\mathcal{D}_{s}\right]$ by the rule

$$
v_{\mathbf{O}}^{(s)}(x, K)=\min _{j \in \mathbf{I}(K)} \min _{z \in \mathbb{A}_{j}(x, K)}\left[\mathbf{c}\left(x, \operatorname{pr}_{1}(z), K\right)+c_{j}(z, K)+v_{\mathbf{O}}^{(s-1)}\left(\operatorname{pr}_{2}(z), K \backslash\{j\}\right)\right] \quad \forall(x, K) \in D_{s} .
$$

If $s=N$, then $\hat{V}[x \mid \mathbf{O}]=v_{\mathbf{O}}^{(s)}(x, \overline{1, N}) \quad \forall x \in X^{0}$ (we obtain function (5.3)); our procedure is complete. If $s<N$, then $v_{\mathbf{O}}^{(s-1)}$ is annihilated and replaced by $v_{\mathbf{O}}^{(s)}$. The layer $v_{\mathbf{O}}^{(s)}$ is used for constructing $v_{\mathbf{O}}^{(s+1)}$. As a result, we obtain $v_{\mathbf{O}}^{(N)} \in \mathcal{R}_{+}\left[\mathcal{D}_{N}\right]$ for which

$$
\hat{V}[\cdot \mid \mathbf{O}]=v_{\mathbf{O}}^{(N)} .
$$

Further, we use (5.5) to construct a majorizing function on $X^{0}$. In addition, by (2.16) and (3.2) for $x \in X^{0}, \alpha \in \mathbb{P}$, and $\left(z_{t}\right)_{t \in \overline{0, N}} \in \mathcal{Z}_{\alpha}[x]$, we have

$$
\mathfrak{C}_{\alpha}\left[\left(z_{t}\right)_{t \in \overline{0, N}} \mid x\right]=\hat{\mathfrak{C}}_{\alpha}\left[\left(z_{t}\right)_{t \in \overline{0, N}} \mid \mathbf{O}\right]+f\left(\operatorname{pr}_{2}\left(z_{N}\right), x\right) \geq \hat{\mathfrak{C}}_{\alpha}\left[\left(z_{t}\right)_{t \in \overline{0, N}} \mid \mathbf{O}\right] .
$$

As a result, from (2.17), (3.3), and (5.6), we get that

$$
\hat{V}[x \mid \mathbf{O}] \leq V[x] \quad \forall x \in X^{0} .
$$

So, for now, we have a lower bound for $V[\cdot]$. To construct an upper bound, we need a procedure of type (3.26)-(3.30). First, we implement the construction procedure for all functions

$$
v_{\mathbf{O}}^{(0)}, \quad v_{\mathbf{O}}^{(1)}, \quad \ldots, \quad v_{\mathbf{O}}^{(N)}
$$

using the above variant from the present section with the following correction: all functions (5.8) are preserved in computer memory. So, we do not rewrite layers of the Bellman function. 
As a result, we obtain all layers (5.8). It is important that all these layers are constructed by one DP procedure: we have a DP procedure universal relative to the starting point. As in Section 4, this procedure is based on (5.4). But in this procedure, we accumulate our own knowledge about (5.8) (when defining only (5.5), we do not accumulate this knowledge).

Further, by means of layers (5.8), for every $x \in X^{0}$, we use (4.8)-(4.11) for $\mathbf{f}=\mathbf{O}$. So, for every $x \in X^{0}$, we find the set $\widehat{\mathbb{S}}[x \mid \mathbf{O}]$ (see (3.6)) of all optimal solutions to problem (3.3).

Further, for $x \in X^{0}$ and $(\alpha, \mathbf{z}) \in \hat{\mathbb{S}}[x \mid \mathbf{O}]$, we set

$$
\tilde{v}(\alpha, \mathbf{z}, x) \triangleq \hat{V}[x \mid \mathbf{O}]+f\left(\operatorname{pr}_{2}(\mathbf{z}(N)), x\right) .
$$

We obtain the following new dependence for $x \in X^{0}$ :

$$
(\alpha, \mathbf{z}) \longmapsto \tilde{v}(\alpha, \mathbf{z}, x): \hat{\mathbb{S}}[x \mid \mathbf{O}] \longrightarrow \mathbb{R}_{+} .
$$

Consider the number

$$
\hat{\mathbb{V}}[x] \triangleq \min _{(\alpha, \mathbf{z}) \in \hat{\mathbb{S}}[x \mid \mathbf{O}]} \tilde{v}(\alpha, \mathbf{z}, x)=\hat{V}[x \mid \mathbf{O}]+\min _{(\alpha, \mathbf{z}) \in \hat{\mathbb{S}}[x \mid \mathbf{O}]} f\left(\operatorname{pr}_{2}(\mathbf{z}(N)), x\right) .
$$

Of course, (5.10) defines a function $\hat{\mathbb{V}}[\cdot], x \longmapsto \hat{\mathbb{V}}[x]: X^{0} \longrightarrow \mathbb{R}_{+}$.

Proposition 1. If $x \in X^{0}$, then $V[x] \leq \tilde{v}(\alpha, \mathbf{z}, x) \quad \forall(\alpha, \mathbf{z}) \in \hat{\mathbb{S}}[x \mid \mathbf{O}]$.

P r o o f. Fix $x^{*} \in X^{0}$ and $\left(\alpha^{*}, \mathbf{z}^{*}\right) \in \hat{\mathbb{S}}[x \mid \mathbf{O}]$. Then, by (5.9), we have

$$
\tilde{v}\left(\alpha^{*}, \mathbf{z}^{*}, x^{*}\right)=\hat{V}\left[x^{*} \mid \mathbf{O}\right]+f\left(\operatorname{pr}_{2}\left(\mathbf{z}^{*}(N)\right), x^{*}\right) .
$$

In addition, by (3.2) and (5.1), we obtain the equality

$$
\hat{\mathfrak{C}}_{\alpha^{*}}\left[\mathbf{z}^{*} \mid \mathbf{O}\right]=\sum_{t=1}^{N}\left[\mathbf{c}\left(\operatorname{pr}_{2}\left(\mathbf{z}^{*}(t-1)\right), \operatorname{pr}_{1}\left(\mathbf{z}^{*}(t)\right),\left\{\alpha^{*}(k): k \in \overline{t, N}\right\}\right)+c_{\alpha^{*}(t)}\left(\mathbf{z}^{*}(t),\left\{\alpha^{*}(k): k \in \overline{t, N}\right\}\right)\right] .
$$

By the choice of $\left(\alpha^{*}, \mathbf{z}^{*}\right)$ and (3.6), the following equality holds:

$$
\hat{\mathfrak{C}}_{\alpha^{*}}\left[\mathbf{z}^{*} \mid \mathbf{O}\right]=\hat{V}\left[x^{*} \mid \mathbf{O}\right] .
$$

By (2.16) and (5.11)-(5.12), we get that

$$
\begin{aligned}
\tilde{v}\left(\alpha^{*}, \mathbf{z}^{*}, x^{*}\right)= & \hat{\mathfrak{C}}_{\alpha^{*}}\left[\mathbf{z}^{*} \mid \mathbf{O}\right]+f\left(\operatorname{pr}_{2}\left(\mathbf{z}^{*}(N)\right), x^{*}\right)=\sum_{t=1}^{N}\left[\mathbf{c}\left(\operatorname{pr}_{2}\left(\mathbf{z}^{*}(t-1)\right) \operatorname{pr}_{1}\left(\mathbf{z}^{*}(t)\right),\left\{\alpha^{*}(k): k \in \overline{t, N}\right\}\right)\right. \\
& \left.+c_{\alpha^{*}(t)}\left(\mathbf{z}^{*}(t),\left\{\alpha^{*}(k): k \in \overline{t, N}\right\}\right)\right]+f\left(\operatorname{pr}_{2}\left(\mathbf{z}^{*}(N)\right), x^{*}\right)=\mathfrak{C}_{\alpha^{*}}\left[\mathbf{z}^{*} \mid x^{*}\right] .
\end{aligned}
$$

In addition, $\left(\alpha^{*}, \mathbf{z}^{*}\right) \in \tilde{D}\left[x^{*}\right]$ (see (3.6)). Therefore (see (2.17)), $V\left[x^{*}\right] \leq \mathfrak{C}_{\alpha^{*}}\left[\mathbf{z}^{*} \mid x^{*}\right]$. The required inequality $\tilde{v}\left(\alpha^{*}, \mathbf{z}^{*}, x^{*}\right) \geq V\left[x^{*}\right]$ follows from (5.13). Since $x^{*}$ and $\left(\alpha^{*}, \mathbf{z}^{*}\right)$ were chosen arbitrarily, our proposition is established.

Corollary 1. The inequality $V[x] \leq \hat{\mathbb{V}}[x]$ holds for $x \in X^{0}$.

P r o o f. The proof is an immediate combination of (5.10) and Proposition 1.

So, we get that $\hat{\mathbb{V}}[\cdot] \triangleq(\hat{\mathbb{V}}[x])_{x \in X^{0}} \in \mathcal{R}_{+}\left[X^{0}\right]$ is a majorant for $V[\cdot]$ :

$$
V[x] \leq \hat{\mathbb{V}}[x] \quad \forall x \in X^{0} .
$$


In this connection, we introduce the number

$$
\mathbf{V} \triangleq \min _{x \in X^{0}} \hat{\mathbb{V}}[x] \in \mathbb{R}_{+} .
$$

From (2.21), (5.14), and (5.15), we obtain the following estimate:

$$
\mathbb{V} \leq \mathbf{V}
$$

For $x \in X^{0}$, we consider

$$
\tilde{v}(\alpha, \mathbf{z}, x) \longrightarrow \min , \quad(\alpha, \mathbf{z}) \in \hat{\mathbb{S}}[x \mid \mathbf{O}],
$$

as a majorant problem. Now, we consider a variant of a minorant problem. In this construction, we aim to make (5.7) more precise. For this, we introduce the function

$$
\left.\left.\varphi \triangleq\left(\min _{y \in X^{0}} f(x, y)\right)_{x \in \mathbf{M}} \in \mathcal{R}_{+}\right] \mathbf{M}\right]
$$

Now, we consider the scheme from Section 3 for $\mathbf{f}=\varphi$. In this case, we implement layers (3.21); as a result, we obtain the functions

$$
v_{\varphi}^{(0)} \in \mathcal{R}_{+}\left[\mathcal{D}_{0}\right], \quad v_{\varphi}^{(1)} \in \mathcal{R}_{+}\left[\mathcal{D}_{1}\right], \quad \ldots, \quad v_{\varphi}^{(N)} \in \mathcal{R}_{+}\left[\mathcal{D}_{N}\right] .
$$

We have a successive implementation $v_{\varphi}^{(0)} \longrightarrow v_{\varphi}^{(1)} \longrightarrow \cdots \longrightarrow v_{\varphi}^{(N)}$. In addition,

$$
v_{\varphi}^{(0)}(x, \varnothing)=\varphi(x) \quad \forall x \in \bigcup_{i \in \overline{1, N} \backslash \mathbf{K}_{1}} \mathbf{M}_{i} .
$$

If $s \in \overline{1, N}$, then the transformation of $v_{\varphi}^{(s-1)}$ to $v_{\varphi}^{(s)}$ is defined by (3.24) for $\mathbf{f}=\varphi$. In this construction, we use a procedure with rewriting layers: for $s<N$, we use $v_{\varphi}^{(s-1)}$ to construct $v_{\varphi}^{(s)}$ by the above variant of (3.24) and annihilate $v_{\varphi}^{(s-1)}$ thereafter; using $v_{\varphi}^{(s)}$, we construct $v_{\varphi}^{(s+1)}$ and so on. From (3.25), we get that

$$
\hat{V}[x \mid \varphi]=v_{\varphi}(x, \overline{1, N})=v_{\varphi}^{(N)}(x, \overline{1, N}) \quad \forall x \in X^{0} .
$$

By (5.17), we obtain the function $\hat{V}[\cdot \mid \varphi]$. Now, by analogy with [6], we introduce an estimating set

$$
X_{0} \triangleq\left\{x \in X^{0} \mid \hat{V}[x \mid \varphi] \leq \mathbf{V}\right\} .
$$

The set $X_{\mathrm{opt}}^{0} \triangleq\left\{x \in X^{0} \mid V[x]=\mathbb{V}\right\}$ satisfies the inclusion

$$
X_{\mathrm{opt}}^{0} \subset X_{0} \text {. }
$$

The proof is similar to that of [6, Proposition 5.1].

Remark 2. For completeness, we verify (5.19). Let $y \in X_{\mathrm{opt}}^{0}$. Then $y \in X^{0}$ and $V[y]=\mathbb{V}$. Using (2.18), we choose $\left(\alpha^{\prime}, \mathbf{z}^{\prime}\right) \in(\mathrm{SOL})[y]$. By the choice of $y$, we have

$$
\mathfrak{C}_{\alpha^{\prime}}\left[\mathbf{z}^{\prime} \mid y\right]=V[y]=\mathbb{V} .
$$

In addition, $\varphi\left(\operatorname{pr}_{2}\left(\mathbf{z}^{\prime}(N)\right)\right) \leq f\left(\operatorname{pr}_{2}\left(\mathbf{z}^{\prime}(N), y\right)\right.$. As a corollary, by (3.18) and (3.2), we obtain

$$
\begin{aligned}
& \hat{\mathfrak{C}}_{\alpha^{\prime}}\left[\mathbf{z}^{\prime} \mid \varphi\right]=\sum_{t=1}^{N}\left[\mathbf{c}\left(\operatorname{pr}_{2}\left(\mathbf{z}^{\prime}(t-1)\right), \operatorname{pr}_{1}\left(\mathbf{z}^{\prime}(t)\right),\left\{\alpha^{\prime}(k): k \in \overline{t, N}\right\}\right)\right. \\
& \left.+c_{\alpha^{\prime}(t)}\left(\mathbf{z}^{\prime}(t),\left\{\alpha^{\prime}(k): k \in \overline{t, N}\right\}\right)\right]+\varphi\left(\operatorname{pr}_{2}\left(\mathbf{z}^{\prime}(N)\right) \leq \mathfrak{C}_{\alpha^{\prime}}\left[\mathbf{z}^{\prime} \mid y\right] .\right.
\end{aligned}
$$


Since $\left(\alpha^{\prime}, \mathbf{z}^{\prime}\right) \in \tilde{D}[y]$, we get that $\hat{V}[y \mid \varphi] \leq \hat{\mathfrak{C}}_{\alpha^{\prime}}\left[\mathbf{z}^{\prime} \mid \varphi\right]$. By (5.21), $\hat{V}[y \mid \varphi] \leq \mathfrak{C}_{\alpha^{\prime}}\left[\mathbf{z}^{\prime} \mid y\right]$. Using (5.16) and (5.20), we get that

$$
\hat{V}[y \mid \varphi] \leq \mathbf{V}
$$

By (5.18) and (5.22), the inclusion $y \in X_{0}$ holds. Since $y$ was chosen arbitrarily, the inclusion (5.19) is established.

Note that $X_{\mathrm{opt}}^{0} \neq \varnothing$ (see (2.21)) and, as a corollary, according to (5.19), $X_{0} \neq \varnothing$. So, $X_{0} \in \mathcal{P}^{\prime}\left(X^{0}\right)$; by the definition from Section 2 ,

$$
\mathbb{V}=\min _{x \in X_{0}} V[x] .
$$

Now, by analogy with [6, Sect. 5], we obtain the following scheme for solving problem (2.19). Let us list basic steps.

(1) Determine the upper bound $\mathbf{V}(5.15)$ by solving the majorizing problem by means of a universal (relative to points from $X^{0}$ ) variant of DP.

(2) Solve the minorating problem constructing $\hat{V}[\cdot \mid \varphi]=v_{\varphi}^{(N)}$ by the scheme with rewriting layers of the Bellman function (it is a universal (relative to points from $X^{0}$ ) variant of DP).

(3) Construct the set $X_{0}$.

(4) Solve all $x$-problems (2.17), $x \in X_{0}$, by means of individual variants of DP; for this, determine $V[x], x \in X_{0}$, and implement $\mathbb{V}$ by (5.23) is effective. Moreover, for the obtained point $x^{0} \in X_{\mathrm{opt}}^{0}$, by a procedure similar to (4.8)-(4.11), an $x^{0}$-optimal solution $\left(\alpha^{0},\left(z_{t}^{0}\right)_{t \in \overline{0, N}}\right) \in \tilde{D}\left[x^{0}\right]$ is found. As a result, $\left(\alpha^{0},\left(z_{t}^{0}\right)_{t \in \overline{0, N}}, x^{0}\right) \in$ SOL.

Note that step (4) can be implemented as follows. First, for every $x \in X_{0}$, we implement a DP procedure with rewriting layers of the Bellman function (we keep in mind an individual DP procedure). As a result, we obtain $V[x], x \in X_{0}$. Further, we find $x^{0} \in X_{0}$ for which (see (5.23)) $V\left[x^{0}\right]=\mathbb{V}$ (more precisely, we find the point of minimum of $V[x], x \in X_{0}$ ). Then, $x^{0} \in X_{\mathrm{opt}}^{0}$. After that, we implement an individual variant of DP for the case $x=x^{0}$ (see (4.8)-(4.12)).

\section{Weakening of the closed routing problem}

In this section, we will take a very short look at one traditional variant of the closed routing problem, as well as its natural weakening. In our construction, we are oriented to [6, Sect. 6]. Let $\rho \in \mathcal{R}_{+}[X \times X]$ be a metric on the set $X$. So, $(X, \rho)$ is a metric space. Suppose that

$$
f(\tilde{x}, x)=\rho(\tilde{x}, x) \quad \forall \tilde{x} \in \mathbf{M} \quad \forall x \in X^{0} .
$$

In fact, this requirement means that we consider the routing problem with return to the starting point (see (2.16)) and the latter means the distance (this interpretation is more natural for metric routing problems where other components of additive criterion mean the distance). In this connection, we recall the known closed TSP (see [7, 11]).

Remark 3. Note that, in the general setting in Section 2, we can consider solutions with terminal permutation to the starting point.

In applied problems, the requirement of return can often be weakened. Consider one variant of such weakening. Let

$$
B_{\rho}^{0}(x, \varepsilon) \triangleq\left\{y \in X^{0} \mid \rho(x, y) \leq \varepsilon\right\}
$$


for $x \in X^{0}$ and $\varepsilon \in \mathbb{R}_{+}, \varepsilon>0$. We fix this number $\varepsilon, \varepsilon>0$. In what follows, we replace $f(6.1)$ using another definition. For $x \in X$ and $A \in \mathcal{P}^{\prime}(X)$, let $\rho(x ; A) \triangleq \inf (\{\rho(x, y): y \in A\}) ; \rho(x ; A) \in \mathbb{R}_{+}$. Suppose that

$$
f(\tilde{x}, x) \triangleq \rho\left(\tilde{x} ; B_{\rho}^{0}(x, \varepsilon)\right) \quad \forall \tilde{x} \in \mathbf{M} \quad \forall x \in X^{0} .
$$

Recall that $\varphi \in \mathcal{R}_{+}[\mathbf{M}]$ is defined by the rule

$$
\varphi(x)=\min _{y \in X^{0}} f(x, y)=\min _{y \in X^{0}} \rho\left(x ; B_{\rho}^{0}(y, \varepsilon)\right) \quad \forall x \in \mathbf{M} .
$$

Proposition 2. The equality $\varphi(x)=\rho\left(x ; X^{0}\right)$ holds for $x \in \mathbf{M}$ in the case (6.2).

P r o o f. The corresponding scheme is similar to [6, Proposition 6.1]. Let us describe it for completeness. Fix $x_{*} \in \mathbf{M}$. Then

$$
\rho\left(x_{*} ; X^{0}\right)=\min _{y \in X^{0}} \rho\left(x_{*}, y\right)
$$

For every $y \in X^{0}$, the inclusion $\left.y \in B_{\rho}^{0}(y, \varepsilon)\right)$ holds; therefore, by (6.3), we have the inequality $f\left(x_{*}, y\right) \leq \rho\left(x_{*}, y\right)$. As a result, $\varphi\left(x_{*}\right) \leq \rho\left(x_{*}, y\right)$ for $y \in X^{0}$. Therefore, by (6.4), we have

$$
\varphi\left(x_{*}\right) \leq \rho\left(x_{*} ; X^{0}\right) .
$$

Since $\left.B_{\rho}^{0}(y, \varepsilon)\right) \subset X^{0}$, we have $\rho\left(x_{*} ; X^{0}\right) \leq \rho\left(x_{*} ; B_{\rho}^{0}(y, \varepsilon)\right)=f\left(x_{*}, y\right)$ for $y \in X^{0}$. In view of (6.3), the inequality $\rho\left(x_{*} ; X^{0}\right) \leq \varphi\left(x_{*}\right)$ is established. Using (6.5), we obtain the equality $\varphi\left(x_{*}\right)=\rho\left(x_{*} ; X^{0}\right)$. Since $x_{*}$ was chosen arbitrarily, the required statement is obtained.

So, we get that (in our case) $\varphi=\left(\rho\left(x ; X^{0}\right)\right)_{x \in \mathbf{M}}$ and our minorant problem coincides with the simplest variant of Consider the simplest version of the problem that implements the lower estimate. But, using (6.2) (instead of (6.1)), we decrease $\mathbf{V}$ somewhat with respect to (6.1). Indeed, $\rho\left(\tilde{x} ; B_{\rho}^{0}(x, \varepsilon)\right) \leq \rho(\tilde{x}, x)$ for $\tilde{x} \in \mathbf{M}$ and $x \in X^{0}$.

We can consider the replacement $(6.1) \longrightarrow(6.2)$ as a weakening of the initial problem with the terminal function (6.1). For this weakening, it is required to successfully achieve a "reduction" of the set $X_{0}$ compared to $X^{0}$.

\section{Computational experiment}

In this section, we will consider examples related to the engineering problem of cutting sheets on CNC machines. It is assumed that a sheet cutting plan already exists. More precisely, cutting should be done along the equidistances of the contours. For each equidistance, a corresponding sampling is made. So, in fact, we have a "discrete equidistance." For each point of such a discrete equidistance, from the outside to the part, there are a piercing point and a switch-off point of the tool. Besides, each pair of these points corresponds to the starting point of the contour cut.

We consider the set of all such points as a megacity (we mean the set of all points of the two types listed above). The tool moves to the piercing point in idle (fast) mode. Upon reaching this point, the tool enters the working mode and begins to pierce the metal. After piercing, the tool moves in cutting mode to the starting point of the contour cutting. It then performs a contour cut with finishing at the start point of the cut and travels to the switch-off point, where the tool shuts off and starts idling to the next contour of the finish point. We do not affect the contour cut. Each contour cut must be made once. So, we exclude this process from consideration. But 


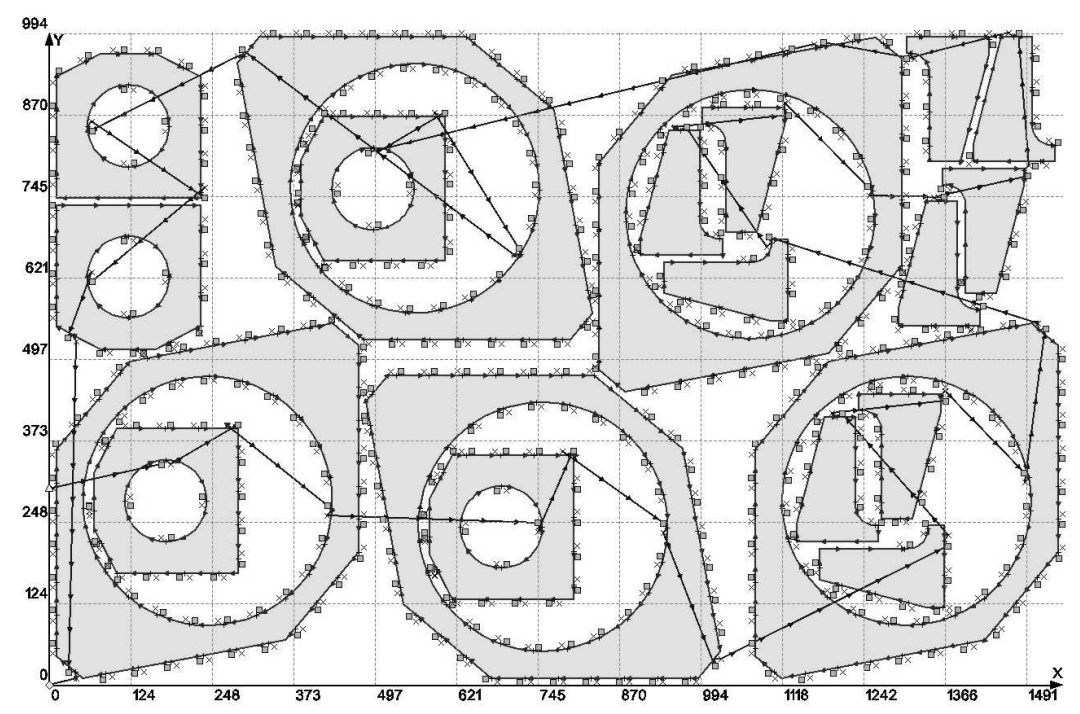

Figure 1. Calculation results for Example 1.

other movements can be chosen to minimize the overall tool time. It is required to regulate the visiting process. Besides, we have to select a sequence of piercing points (and the corresponding contour cut start points and tool switch-off points). Finally, we must choose a starting point for our process.

Let us recall the restrictions. The precedence condition (in particular) is associated with the following requirement: cutting the inner contours must precede the outer ones (there are other options). In connection with other restrictions, we note the thermal tolerance (these restrictions will be considered in the second example). These constraints lead to the use of task-list-dependent cost functions (in our model).

In the first example, we will investigate the case considered in Section 3 (see (3.1)). Define the sets

$$
A_{j}(x, K), \quad j \in \overline{1, N}, \quad x \in \mathbf{X} \backslash \mathbf{M}_{j}, \quad K \in \mathfrak{N},
$$

for reasons of maximin of the Euqlidian distance with respect to the cut out contours. In the second example, we will suppose that $A_{j}(x, K)=\mathfrak{M}_{j}$ for all $j \in \overline{1, N}, x \in \mathbf{X} \backslash \mathbf{M}_{j}$, and $K \in \mathfrak{N}^{(j)}$ (so, here we can select any piercing point).

Example 1. The calculations for the first example were performed on a computer with an Intel Xeon CPU E5-2620 processor, 8 GB of memory, and a Windows 10 (64-bit) operating system. The program was developed in $\mathrm{C}++$ using the Qt library to build a user interface.

The number of contours is 30 . The number of ordered pairs is 20 .

The starting point was chosen from a rectangle with corners $(0 \mathrm{~mm}, 0 \mathrm{~mm}),(0 \mathrm{~mm}, 1000 \mathrm{~mm})$, $(1550 \mathrm{~mm}, 1000 \mathrm{~mm})$, and $(1550 \mathrm{~mm}, 0 \mathrm{~mm})$. The step of point checking was $100 \mathrm{~mm}$.

The result is 74.507 . The starting point is $(0 \mathrm{~mm}, 300 \mathrm{~mm})$. The terminal point is $(0 \mathrm{~mm}, 0 \mathrm{~mm})$. The duration of calculation is $32 \mathrm{~h} 29$ min $55 \mathrm{sec}$. The counting results are shown in Fig. 1.

Example 2. This example used a computer with an Intel i7-2630QM processor, 8 GB of memory, and a Windows 7 (64-bit) operating system. The same language and libraries were used.

This example uses the cost functions from [5]. These functions are dependent on the list of visited megacities and are related to the technical limitations of CNC metal cutting plants. This allows thermal restrictions to be taken into account. There should be enough metal to provide a quality of the cut around the finish cut segment. The dependence on the task list allows us to 


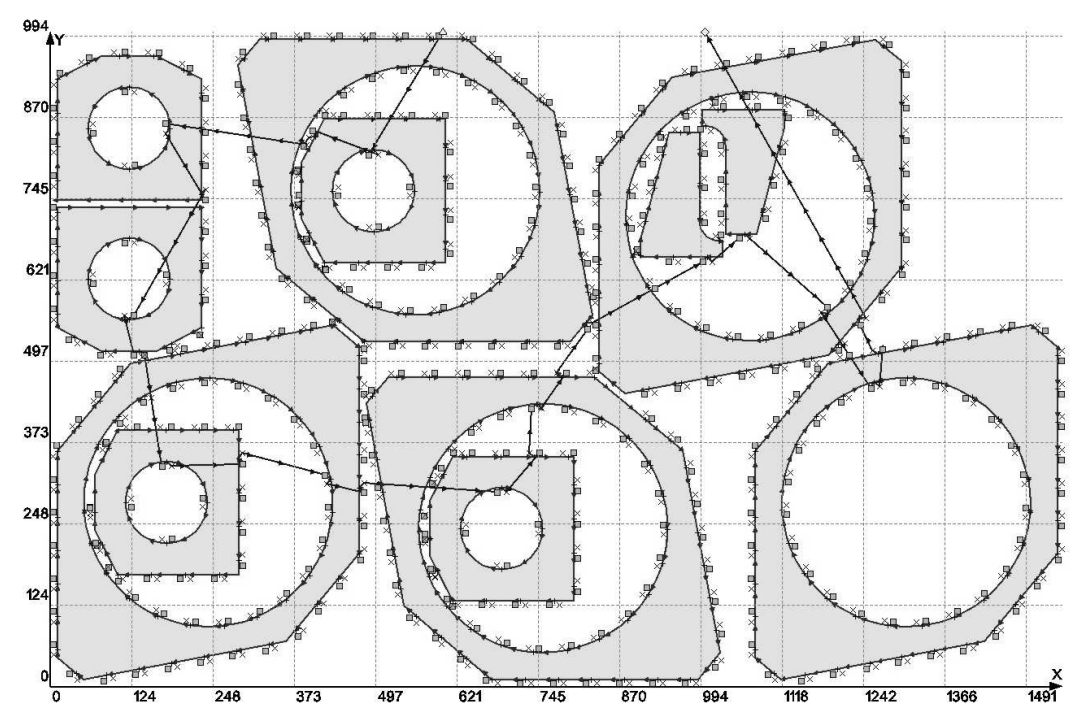

Figure 2. Calculation results for Example 2.

account for the cut-out contours to fix the voids in the metal located near the sections of the cut completion.

The length of the finish cut area $300 \mathrm{~mm}$ (see [5]). The width of the finish cut area is $150 \mathrm{~mm}$. A 1,000,000 penalty was used if $25 \%$ (or more) of the finish cut area was covered with holes in the metal or outside the sheet space.

The starting point was chosen from a rectangle with corners $(0 \mathrm{~mm}, 0 \mathrm{~mm}),(0 \mathrm{~mm}, 1000 \mathrm{~mm})$, $(1550 \mathrm{~mm}, 1000 \mathrm{~mm})$, and $(1550 \mathrm{~mm}, 0 \mathrm{~mm})$. The step of point checking was $100 \mathrm{~mm}$. Starting and finishing points can be different. The maximum range from start to finish point must be less than $500 \mathrm{~mm}$.

First, a count of all start-finish points was made. 51 calculations were made. The obtained result is 50,145 . The start point is $(600 \mathrm{~mm}, 1000 \mathrm{~mm})$, and the finish point is $(1000 \mathrm{~mm}, 1000 \mathrm{~mm})$. The duration of the calculations is $34 \mathrm{~min} 52 \mathrm{sec}$. The results are shown in Fig. 2.

Then the upper estimate of the result was found with a value of 50475 . The calculation time was $40 \mathrm{sec}$. The number of start-finish points was reduced from 51 to 34 . For these 34 points, calculations were made with a counting time of $23 \mathrm{~min} 5 \mathrm{sec}$. The result obtained, of course, is the same as in the case of using all start-finish points. So, the total time spent on the process with counting the estimates and reducing the number of points is $24 \mathrm{~min} 25 \mathrm{sec}$. This is less than the total calculation time for 51 points (34 min $52 \mathrm{sec}$ ).

\section{Conclusion}

The paper discusses an "additive" routing problem with constraints and cost functions. depending on the task list. The well-known DP solution method is applied. The optimal choice of the starting point, route, and specific trajectory has been implemented. The settings are investigated with the requirement to return to a neighborhood of the starting point and without this requirement. Thus, individual (to the starting point) and universal (relative to the starting point) DP procedures arise. In addition, the option of using a universal DP is proposed for the application of an individual DP procedure. 


\section{REFERENCES}

1. Bellman R. Dynamic programming treatment of the travelling salesman problem. J. ACM, 1962. Vol. 9, No. 1. P. 61-63. DOI: 10.1145/321105.321111

2. Chentsov A.A., Chentsov A. G. Routization problem complicated by the dependence of costs functions and "current" restrictions from the tasks list. Model. Anal. Inf. Sist., 2016. Vol. 23, No. 2. P. 211-227. DOI: 10.18255/1818-1015-2016-2-211-227 (in Russian)

3. Chentsov A. G. Ekstremal'nye zadachi marshrutizacii i raspredeleniya zadanij: voprosy teorii [Extreme routing and distribution tasks: theory questions]. M.-Izhevsk: R\&C Dynamics. Izhevsk Institute of Computer Research, 2008. 240 p. (in Russian)

4. Chentsov A. G. To question of routing of works complexes. Vestn. Udmurtsk. Univ. Mat. Mekh. Komp. Nauki, 2013. No. 1. P. 59-82. (in Russian)

5. Chentsov A. G., Chentsov P. A. Routing under constraints: Problem of visit to megalopolises. Autom. Remote Control, 2016. Vol. 77, No. 11. P. 1957-1974. DOI: 10.1134/S0005117916110060

6. Chentsov A.G., Chentsov P. A. To the question of optimization of the starting point in the routing problem with restrictions. Izv. IMI UdGU, 2020. Vol. 55. P. 135-154. DOI: 10.35634/2226-3594-2020-55-09 (in Russian)

7. Cook W. J. In Pursuit of the Traveling Salesman. Mathematics at the Limits of Computation. N. J.: Princeton Univer. Press, 2012. 272 p. https://www.jstor.org/stable/j.ctt7t8kc

8. Dieudonné J. Foundations of Modern Analysis. New York: Academic Press, 1960. 361 p.

9. Held M., Karp R.M. A dynamic programming approach to sequencing problems J. Soc. Indust. Appl. Math., 1962. Vol. 10, No. 1. P. 196-210. DOI: 10.1137/0110015

10. Gimadi E. Kh., Khachay M. Ekstremal'nye zadachi na mnozhestvah perestanovok [Extremal Problems on Sets of Permutations]. Ekaterinburg: Izdatel'stvo UMC UPI, 2016. 220 p. (in Russian)

11. Gutin G., Punnen A.P. The Traveling Salesman Problem and Its Variations. Boston: Springer, 2007. 830 p. DOI: $10.1007 / \mathrm{b} 101971$

12. Little J. D. C., Murty K. G., Sweeney D. W., Karel C. An algorithm for the traveling salesman problem. Oper. Res., 1963. Vol. 11, No. 6. P. 972-989. DOI: 10.1287/opre.11.6.972

13. Kosheleva M.S., Chentsov A.A., Chentsov A. G. On a routing problem with constraints that include dependence on a task list. Trudy Inst. Mat. i Mekh. UrO RAN, 2015. Vol. 21, No. 4. P. 178-195. (in Russian)

14. Kuratowski K., Mostowski A. Set Theory. North-Holland, 1968. 417 p.

15. Lawler E.L. Efficient Implementation of Dynamic Programming Algorithms for Sequencing Problems. CWI. Technical Reports. Stichting Mathematish Centrum. Mathematische Besliskunde, 1979. BW 106/79. 16 p.

16. Melamed I. I., Sergeev S. I., Sigal I. Kh. The traveling salesman problem. I: Issues in theory. Autom. Remote Control, 1989. Vol. 50, No. 9. P. 1147-1173.

17. Melamed I. I., Sergeev S. I., Sigal I. Kh. The traveling salesman problem. II: Exact methods. Autom. Remote Control, 1989. Vol. 50, No. 10. P. 1303-1324.

18. Melamed I. I., Sergeev S. I., Sigal I. Kh. The traveling salesman problem. Approximate algorithms. Autom. Remote Control, 1989. Vol. 50, No. 11. P. 1459-1479. 